\title{
Access of Distributed Generation and Its Energy Management System Development
}

\author{
B.Zeng \\ Guangxi Power Grid Electric Power Research Institute \\ Nanning, China
}

\author{
X.Y. Kong, Q.A. Hu, S.Y. Zhang, Q. Yang, S.Y. Ge \\ Key Laboratory of Smart Grid of Ministry of Education \\ Tianjin University \\ China
}

\begin{abstract}
The access of distributed generation is one of the important functions for future energy management system. In this paper, technologies with information interaction of devices and energy management system for distributed generation "plug and play" access was analyzed. A "plug and play" control model and its application energy management system were introduced. The proposed system could meet power system connection requirements and operation limitations by guaranteeing the output characteristic of distributed generation. It will provide a reference for future corresponding study.
\end{abstract}

Keywords-distributed generation; plug and play; energy management system; smart grid

\section{INTRODUCTION}

One of the core content of the smart grid is to solve the problem of new energy resources access to distributed generation (DG) and keep them security r, reliable and stable operation. Smart grid can simplify the process of the networking. It also allows many different types of DG to access to the system, which similar to the "Plug and play". This feature puts forward a great challenge for the grid. The improved standards of the interconnection can be easily accessed by the generation system and the storage system. All the DGs could be interconnected in all voltage levels, including the photovoltaic power, wind power, and the advanced battery system, plug-in hybrid electric vehicle and the fuel cell. This characteristic is a key factor for the development of the smart grid[1].

The "Plug and play" access of the distributed generation is mainly divided into two schemes: (1) The "plug and play" access of the isolated DG based on the inverter itself; (2) The "plug and play" access of the multiple DGs based on the community energy management system.

The former one can weaken or eliminate the bad effects brought by the DG inverter, it is a best solution, and has a good prospect. But at present due to the standards and the development of the inverter was restricted, it is very difficult to achieve in a short time. The latter one solves the problem through the micro gird, it has a lot of advantages, and the transient problem could be defused in the micro grid when the DG connected to the smart gird. This scheme wasn't a real meaning of "plug and play", it just eliminates the effects of the multiple DGs by the gird energy controller in the micro grid.

\section{IMPLEMENT THE INTERACTION FUNCTION OF THE PNP DEVICE}

PnP is an industry standard originally, used for automatic processing the installation of PC hardware equipment. It set by Intel and Microsoft combined. PnP made the installation of hardware equipment greatly simplified, the system manager of $\mathrm{PnP}$ can automatically detect the equipment accessed, and automatically assigns the resources needed [2]. USB (Universal Serial Bus) device is based on the idea of PnP and widely used now. The realization of the USB's PnP mainly includes two processes of interaction: (1) the interaction between USB device and the host system;2) the interaction between the USB device and driver.

With the development of PnP technology, the standard gradually extended to the whole network and other specific systems (such as the IOT or power system), it made the equipment of the network or specific systems could be found and controlled. For example, the printer accessed to the network, it's an UPnP (Universal Plug and Play).

UPnP is a simple extension of PnP peripheral pattern, it was designed to support zero configurations, "invisible" connected to the Internet, and the automatic detection to a wide range of manufacturers' equipment. This means that a device can dynamically join a network, to obtain an IP address, notify its function, and to understand the existence and the function of other devices. Based on the UPnP network means forming a network by the UPnP protocol, the basic components include (1) devices: UPnP devices is not only hardware devices but also a kind of software services;2)services: the process of the device executive the request of the controller,can be divided into many small modules, each module was called a service;(3) The control point: The control point firstly is a controller who has the ability to control other equipment, and has the ability to "find" the target in the network. After discovering the target, control points will get the information of the equipment, the associated service list and the description of the related services, and then determine the "source" of the event and call for the control services. 


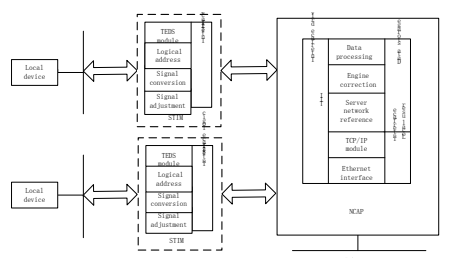

FIGURE I. NETWORK SENSOR MODEL.

International standards organization set some PnP standard for some special equipment[3]. For example, in order to solve the incompatible problem of intelligent sensor products, and implementthe standardization of interface of intelligent sensor underthecondition of the network, IEEE sets interface standard IEEE1451fornetworked intelligent transducer(sensor). The network sensor model is shown in Figure.1.

The goal of the standard is to define a standardized interface between the network and thesensor, it separates the choice of sensors and the choice of network, So the users can choose different smart sensors according to their own needs, and realize the real plug and play.According to the standards of the IEEE1451.1 and IEEE1451.2, network sensor can be divided into two parts,smart transducer interface module STIM and network adapter module NCAP. The STIM follows the standard of IEEE1451.2, by the electronic data table TEDS, 10 lines TII interface and address logic functions, the sensor realizes the function of self-description and the plug and play. One NCAP can connect multiple STIM, so it can realize distributed control easily.

\section{THE SCHEME OF PNP ACCESS OF DistRIBUTED GENERATION}

\section{A. The Principle and Scheme of the System}

When a system has a high number of distributed generations, we can consider the situation that the system under the controlof the community energy management system and other micro power grid control system, we realized the scheme when the distributed generation in the local area network (LAN). In micro grid the DR's built and rebuilt is easily and frequently, which makes the demand of "plug and play" become so important. When we built or rebuilt the DR, the power controller, energy storage, the hardware and software of other control systems and the original system,all the thing can't be changed,Otherwise it will bring us great maintenance costs, and make the degradation ofthe practical of the system[4].

Micro grid's energy management system is an automatic integrated low-pass based on the computer, it combines the advanced IT technology and mainly used for a microgrid dispatching management center, and optimize the management of the energy storage device inside the micro grid. The energy management system of the micro grid has a targeted characteristic when compared with the traditional energy management system of large grid, it can aim at a specific micro grid, do a effective management to the resources inside the grid, and improve the efficiency of the energy use of the micro grid.

As the of micro grid's energy management system, every
DG has its own inverter, they connect to the AC grid through the electrical interfaces, all DGs communicate with the central management unitand are controlled by the unit. When there is a DG access or leave, the central unit will coordinate members according to the sampling signals which obtained from the AC grid, at last eliminate the effects of DG's access or leave.

\section{B. Distributed Generation and Micro Grid Interconnection Interface Design}

In the current design of the distributed generation equipment, the interface of the DG and micro grid as a part of the generation equipment, all manufacturers designed according to their own products, and provide limited information and operation. But for the micro grid, we have more strict requirements for the management of generation unit,mainly reflected in the points as follow:

(1) Distributed generation units need to be plug and play. Due to some renewable energy generation itself has the intermittent features, such as photovoltaic power, wind power, the distributed power may need to frequently incorporated into or cut away from the micro grid system, it needs multiple unit to cooperate to achieve the function, which similar to cooperation of the computer serial port, parallel port and USB port.

(2) All DGs should be brought into the unified scheduling management. In normal situation, DG should adjust its output power under the control of the energy management system or the local control, so that can maintain the stability of the micro grid's operation, economy, etc. In addition, when the whole system is completely cut off, the distributed generation unit needs to help the micro grid to execute the black start, and supply power for important load.

(3) The mode that system supply power for important load must meet the requirements of seamless switching.Considering the power balance principle, Property rights of DGs,electrical distribution, the geographical position and the scope of jurisdiction and scheduling, the independent operation of micro grid can be divided into 2 levels, including the unit island and the whole independent operation.

Interfaces can use the existing products and components (such as switch and circuit breaker, etc.),all these things can be provided by the equipment suppliers. The interface between distributed generation devices and micro grid is independent of the DG andmicro grid, it can be divided into two parts: the power interface and information interface. The power interface connects directly to the micro grid line, information interface connects to the micro grid's center management unit through the communication way, which is the terminal of the DG's management.

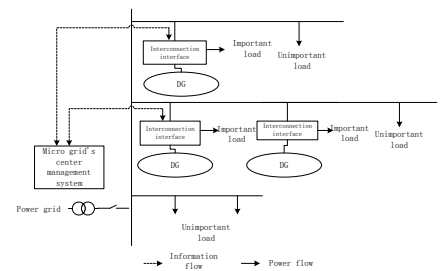

FIGURE II. MULTI-DGSINPOWERGRID. 
Figure. 2 is a figure that multi-DGs connect to the micro grid [5]. When the micro grid contains multiple DGs, the electronic switch of the interconnection interface together with the information interface to realize the PnP, it makes the DG's cut into or from the micro grid system very convenient. Meanwhile, each information interface part could communicate with each other through the central management unit, implement the unified management of the DG's excision, power generation scheduling, fault detection, etc., and help the micro grid to execute the black startwhen the whole system is completely cut off. Important loads access to the system through the power interface, when micro grid and DG operate normally, they supply power for important loads together, either one has fault cases,the system can ensure the power supply of the important loads are not affected, achieve the seamless switching of the power supply.

\section{THE DEVELOPMENT OF THE SYSTEM FUNCTION AND MODULE ANALYSIS}

The "plug and play" access of the isolated DG based on the inverter itselfrequires manufacturers to understand each other's communication protocol to communicate with each other, the early stage of the program has a huge cost, and has some technical difficulties. When the network has excessive amounts of PnP devices, if the communication response is not in time, different devices will repel each other's instructions. So the system took the DG's PnP scheme based on the community energy management system, the energy management system responses to the devices' access and centralized control the equipment's' operation.

The system requires all accessed devices to follow the standard to adjust their communication information, when we set the deployment in the device, it must be fixed in the required range, so that the energy management system can detach the devices"access" and "pull out” [6].

The process of the DG's PnP as follows:

1) Communication server to poll the specified address range, if detach a new device access, validate the device's type.

2) After validatesucceed, the communication server informs the community energy management system.

3) The community energy management system changes the system's space truss structure according to the device's type.

4) The energy management algorithm analysis the energy optimization scheduling according to the new space truss structure, getting a result and distribute in time.

5) Communication server to poll the specified address range, if detach the device removed from the system.

6) The communication server informs the community energy management system to change the space truss structure and remove the device.

7) The energy management algorithm analysis the energy optimization scheduling according to the new space truss structure, getting a result and distribute in time.

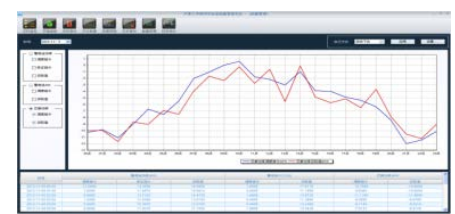

FIGURE III. RESULT OF ENERGY MANAGEMENT PROCESS.

The system adopted a real-time detection and a real-time correction method for the scheduling results, on the basis of the results of energy scheduling, and make a real-time collection for the running parameters information of the equipment, then combined with the result of the scheduling of dispatching and make a real-time correction for the command. So it meets the optimal scheduling and avoids the wastage of the energy storage life by the errors in the forecast data. Solved the problem of scheduling instructions do not tally with the actual situation because of the errors between the predicted and actual, it keeps the system economy optimal and greatly extends the service's life of the energy storage, and it also increases the stability of the system.Result of energy management processes shown as Figure. 3.

\section{CONCLUSION}

Build an open communication architecture, form a PnP environment,and make a networked communication between the grid's components is the basis of the PnP; Set up a unified technical standards, realize the seamless communication between the device and application systems, realizing the function of interoperability between device and device, devices and systems, system and system is the central goal of the energy management.

\section{ACKNOWLEDGEMENT}

X.Y. KONG is the corresponding author.The authors thank for funding of National Science and Technology Support Program(2013BAA01B03).

\section{REFERENCES}

[1] Y. Ji, Q. Ai, D. Xie. Research on Co-Developmental Trend of Distributed Generation and Smart Grid[J].Power System Technology, 2010, 34(12):15-23.

[2] J.H. Guo, Q.H. Xie, W. Huang. Study on the Plug and Play Theory in Micro-grid Based on MAS [J].Power System and Clean Energy,2010,26(1):31-35.

[3] NIKKHAJOEI H, LASSETER R H. Distributed generationinterface to the CERTS microgrid. IEEE Trans on PowerDelivery, 2009, 24 (3): 1598-1608.

[4] Y. Luo, L. Shi.Microgrid Common Information Model Applicable to Plug and Play Feature of Distributed Resources[J].Automation of Electric Power System, 2010, 34(8):97-100.

[5] M.Z. Guo, S.M. Tian, Y. Wang. Study on Distributed Gereration "Plug and Play” based on Photovoltaic/Battery Micro-grid)[C]. 2012 electric power communication management andsmartgridcommunicationstechnologyforumProceeding, 322-382.

[6] McArthur S D J, Davidson E M, Catterson V M, et al. Multi-agent systems for power engineering applications-Part II: technologies, standards, and tools for building multi-agent systems[J]. Power Systems, IEEE Transactions onPowerDelivery, 2007, 22(4): 1753-1759. 